\title{
Validity and reliability of the Swaymeter device for measuring postural sway
}

\author{
Daina L Sturnieks', Ria Arnold and Stephen R Lord
}

\begin{abstract}
Background: This study aimed to examine: 1) Swaymeter concurrent validity in discriminating between young and older adult populations; 2) Swaymeter convergent validity against a forceplate system; and 3) the immediate testretest repeatability of postural sway measures obtained from the Swaymeter.

Methods: Twenty-nine older adults aged 71 to 83 years and 11 young adults aged 22 to 47 years had postural sway measured simultaneously with the Swaymeter and a forceplate for three repeat 30 second trials, under four conditions (floor eyes open, floor eyes closed, foam eyes open, foam eyes closed).

Results: Age-related differences in sway parameters across the four conditions were evident using the Swaymeter. Moderate-to-good correlations were found between Swaymeter and forceplate sway measures across conditions ( $r$ $=0.560-0.865$ ). Good agreement between the Swaymeter and forceplate were found for anteroposterior and mediolateral sway displacement measures (average offset $=6 \mathrm{~mm}$ ). Sway path length measures were longer for the forceplate compared to the Swaymeter (average offset $=376 \mathrm{~mm}$ ), but these data showed good agreement following log-transformation. The Swaymeter was reliable across trials, with intraclass correlation coefficients ranging from 0.654 to 0.944 .
\end{abstract}

Conclusions: The Swaymeter is a reliable tool for assessing postural sway and discriminates between performance of young and older people across multiple sensory conditions.

\section{Background}

The control of standing balance is a task of maintaining the body's centre of mass (COM) within the limits of the base of support, achieved by producing forces on the support surface/s (predominantly under the feet while standing). Excursions of the centre of pressure (COP), the point of application of the ground reaction force, measured by a forceplate has been widely used to represent postural sway, as an index of balance control. However, these measures involve technical devices that can be costly and require processing protocols that can make them unfeasible for many clinics and research facilities.

The need for a simple measure of postural sway exists due to the issue of balance problems and the risk of falls in older people. Increasing age is associated with increases in the magnitude and velocity of postural sway during standing $[1,2]$. Indeed, postural sway has been

\footnotetext{
* Correspondence: d.sturnieks@neura.edu.au
Neuroscience Research Australia, Barker Street, Randwick, NSW, 2031,

* Correspondence: d.sturnieks@neura.edu.au Australia
}

(c) 2011 Sturnieks et al; licensee BioMed Central Ltd. This is an Open Access article distributed under the terms of the Creative Commons Attribution License (http://creativecommons.org/licenses/by/2.0), which permits unrestricted use, distribution, and reproduction in any medium, provided the original work is properly cited.

shown to be a risk factor for falls in numerous older populations (see [3] for review). One study has shown multiple fallers to have $33-46 \%$ greater sway than those who did not experience a fall in the previous 12 months [4]. In a prospective study of 100 adults aged 62-96, the root mean square of the mediolateral COP displacement while standing blindfolded was found to predict those people who fell in a follow-up period of 12 months with a predictive accuracy of $67 \%$ [5]. These studies suggest that measuring postural sway, particularly while sensory information is reduced, can provide an indication of an individual's risk of a future fall.

A low tech Swaymeter was designed to address the needs of clinicians and researchers with limited resources (e.g. no access to forceplates or motion laboratories). It is a useful field test, as it is compact, lightweight, has short administration and data processing time. Unlike other lightweight and easily applied systems, such as accelerometers and gyroscopes, the Swaymeter involves no electronics or computer processing. Thus, assessment can be conducted in a variety of 
community settings and health care facilities. Several research groups have found the Swaymeter to be feasible for use in different populations of young and older people [6-8].

The Swaymeter has been used in numerous studies of balance [6-8] as well as retrospective $[9,10]$ and prospective $[11,12]$ investigations of falls risk in older people. Sway path length or sway displacement measures have been found to discriminate between fallers and non-fallers in each of these studies. For example, a prospective study of 341 community-living older women, postural sway assessed with participants standing on a foam mat with eyes open was a significant and independent risk factor for multiple falls [12]. A companion study [20] examined 136 younger women (aged 20-64 years) and reported significant correlations between Swaymeterrecorded postural sway and age, but did not reveal whether postural sway data discriminated between the young and older subgroups. Furthermore, the immediate (intrasession) reliability of the Swaymeter device has not been reported, nor has it been validated against accepted measures of postural sway.

The purpose of this study was to examine: 1) the concurrent validity of the Swaymeter in discriminating between young and older adults; 2) the convergent validity of the Swaymeter against a forceplate system; and 3) the immediate test-retest repeatability of postural sway measures obtained from the Swaymeter. Older adults were hypothesised to have increased postural sway obtained from the Swaymeter, compared to young adults. It was hypothesised that the Swaymeter would show good-to-excellent agreement with forceplate (COP) data, in addition to acceptable reliability across 3 repeated trials.

\section{Methods}

\section{Participants}

Twenty-nine older adults aged 71 to 83 years (mean 78 \pm 3 ) and 11 young adults aged 22 to 47 years (mean age $33 \pm 9$ years) participated in the study. All were healthy, independent and community-dwelling. Older adults were randomly selected from a database of participants previously involved in a large study of falls risk factors. Young adults were recruited from Institute staff. Exclusion criteria were: neurological; cardiovascular or major musculoskeletal impairments; uncorrected visual or vestibular problems; significant pain or recent injury; poor understanding of the English language; Mini Mental Status Examination [13] score < 24; and unable to walk for $20 \mathrm{~m}$ without a walking aid. The study was approved by The University of New South Wales Human Research Ethics Committee and all participants provided informed consent prior to participation.

\section{Protocol}

Assessments of postural sway were performed in bipedal stance, with eyes open and closed, on a firm or compliant (foam) surface, for a total of 4 conditions: floor with eyes open; floor with eyes closed; foam with eyes open; and foam with eyes closed. Conditions were randomly presented and 3 trials of 30 seconds were conducted per condition, for a total 12 trials per participant. Participants were instructed to stand still and without talking, with feet shoulder-width apart and arms crossed over the chest, while looking ahead and slightly down at a blank wall $1.5 \mathrm{~m}$ away. Participants were offered a seat and rested for at least 1 minute in between trials. Recordings from Swaymeter and forceplate devices were recorded simultaneously throughout each trial. During compliant surface trials, a medium-density foam rubber mat $\left(15 \mathrm{~cm}\right.$ thick, $\left.24 \mathrm{~kg} / \mathrm{m}^{3}\right)$ was placed directly over the forceplate. The mechanical characteristics of the foam were such that it was compressed to $9 \mathrm{~cm}$ when a $50 \mathrm{~kg}$ weight was applied equally across its surface.

\section{Swaymeter}

The Swaymeter recorded displacements of the body in the horizontal plane at waist level. The device consisted of an inflexible 40-cm-long rod with a vertically mounted pen at its end. The rod was mounted on a 20 $\mathrm{cm}$ wide metal plate which was fitted over the participant's lower back (level of the posterior superior iliac spine) by a firm belt so that the rod extended posteriorly. Fitted firmly, the Swaymeter offers 1 degree of freedom between the belt and pen as it is free to move in the pitch plane. The pen recorded participant's postural sway on a sheet of millimeter graph paper, fastened to the top of an adjustable-height table (Figure 1). The sway path length was manually determined as the number of millimetre squares traversed by the pen [14]. The anteroposterior (AP) and mediolateral (ML) peakto-peak sway displacements were also calculated from the extremes of sway length in these two planes, as previously described [14].

\section{Ground Reaction Forces}

Ground reaction forces and computed moments were obtained while participants stood on a calibrated $400 \times$ $600 \mathrm{~mm}$ Kistler force plate (9286A, Kistler Instrumente AG, Winterthur, Switzerland) embedded flush with the laboratory floor. Forceplate data were acquired using a CODAmotion 64 channel analogue interface (Charnwood Dynamics, Leicestershire, UK), sampling at 1000 $\mathrm{Hz}$. COP co-ordinate calculations were performed by CODAmotion V6.66 software. COP data were smoothed with a low-pass Savitzky-Golay filter, polynomial order 3 and frame length of 41 (MATLAB R2009a, The 


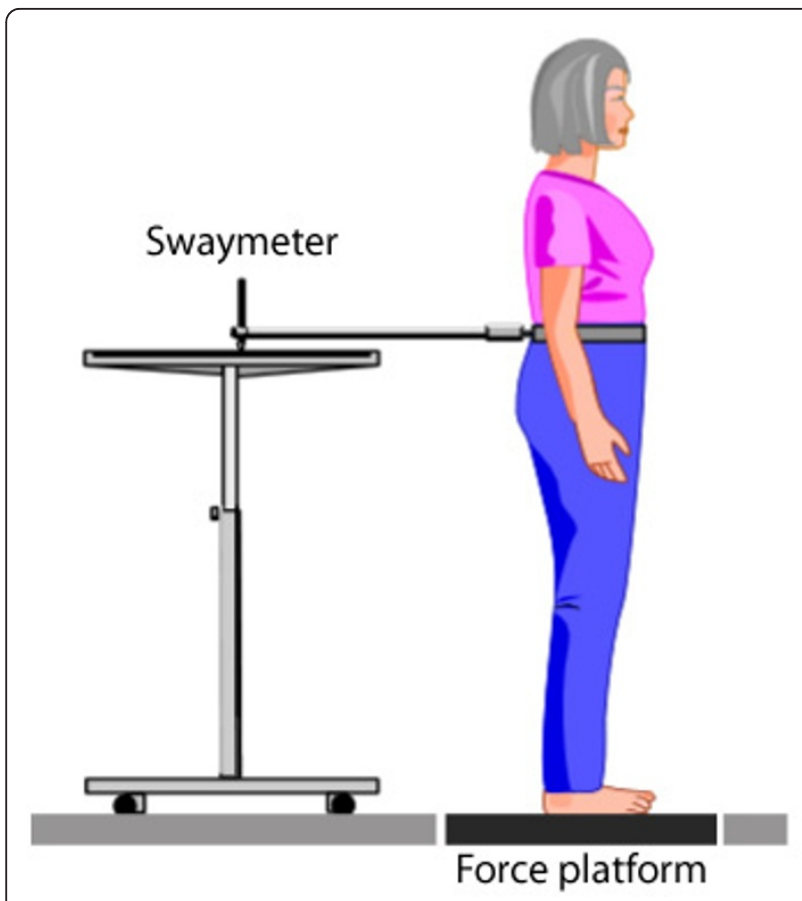

Figure 1 Pictorial representation of the assessment of postural sway using the Swaymeter and forceplate systems.

MathWorks Inc, Massachusetts, USA). COP sway path length was calculated from ML and AP coordinates.

\section{Statistical analysis}

The postural sway variables, calculated from data obtained by the Swaymeter and forceplate, for subsequent analysis were:

1) AP displacement, taken as the maximum position minus the minimum position in the anterioposterior direction;

2) ML displacement, taken as the maximum position minus the minimum position in the mediolateral direction; and

3) Path length, taken as the total sway displacement.

Statistical analyses were performed using SPSS 15.0 for Windows (SPSS Inc, Chicago, USA) with statistical significance set at $\mathrm{p}<0.05$. Descriptive statistics (means and SD) were calculated for each sway variable, across conditions and age groups (young and old). To examine concurrent validity, between-group differences in lognormalised sway variables were examined using 2-factor repeated measures ANOVAs, with group and condition as factors. Bonferonni post-hoc tests were used to identify significant main effects. To examine Swaymeter convergent validity against the forceplate system, Pearson product moment correlation coefficients were calculated for Swaymeter and COP measures across each condition. In addition, offsets and limits of agreement (95\% confidence intervals (CIs)) were calculated according to methods described by Bland and Altman [15]. BlandAltman plots [15] of AP displacement, ML displacement and sway path length during foam eyes open condition were constructed to illustrate Swaymeter agreement with the forceplate system. The foam eyes open condition was chosen as this is most commonly used for clinical and research purposes [16]. To examine the Swaymeter test-retest reliability across 3 repeated trials, intraclass correlation coefficients $(\operatorname{ICC}(2,1))$, 95\% Confidence Intervals (CIs) as well as Standard Error of Means (SEM) were calculated for each condition.

\section{Results}

Data are missing for 2 older adults in the foam eyes closed condition, as they were concerned about their stability and refused to complete the trials. Otherwise, all subjects successfully completed all trials without incident. Table 1 presents participant characteristics and indicates that the study sample comprised relatively healthy groups of young and older people without cognitive impairment.

Descriptive statistics for sway displacement and path length from Swaymeter recordings in the young and older participants are presented in Table 2. Significant group differences existed in Swaymeter variables, with older adults having greater AP displacement, $\left(\mathrm{F}_{1,473}=\right.$ 23.84, $\mathrm{p}<0.0001)$, ML displacement $\left(\mathrm{F}_{1,473}=50.94, \mathrm{p}<\right.$ $0.0001)$ and path length $\left(\mathrm{F}_{1,473}=54.29, \mathrm{p}<0.0001\right)$ than young. Furthermore, there was a significant main effect for condition $\left(\mathrm{F}_{3,473}=53.47, \mathrm{p}<0.0001\right)$, with

Table 1 Anthropometric, fall risk, fall history, health and medical characteristics of young and older participants.

\begin{tabular}{lcc}
\hline Mean (SD) & $\begin{array}{c}\text { Old } \\
\text { (n = 29) }\end{array}$ & $\begin{array}{c}\text { Young } \\
\text { (n = 11) }\end{array}$ \\
\hline Height (cm) & $164.4(9.9)$ & $170.9(6.7)$ \\
Weight (Kg) & $72.5(9.9)$ & $66.3(14.9)$ \\
Physiological Profile Assessment (PPA) falls & $0.60(1.06)$ & - \\
risk score & & \\
Short-form 36 general health score & $70.4(16.9)$ & - \\
(range 0 - 100) & $27.8(1.7)$ & - \\
$\begin{array}{l}\text { Mini Mental Status Examination score } \\
\text { (range 0 - 30) }\end{array}$ & & \\
\hline Number (\%) & $14(48.3)$ & $6(54.5)$ \\
\hline Female gender & $7(24.1)$ & - \\
One or more falls in previous year & $24(82.8)$ & - \\
Two or more medical conditions* & $18(62.1)$ & - \\
Four or more medications & & - \\
\hline
\end{tabular}

*Medical conditions surveyed were; peripheral vascular disease, diabetes, stroke, trans-ischemic attack, heart attack, angina, high blood pressure, heart/ blood vessel problems, and arthritis. 
Table 2 Mean (SD) sway measures ( $\mathrm{mm}$ ) for young and older participants, as determined from Swaymeter recordings.

\begin{tabular}{lcccccccc}
\hline & \multicolumn{3}{c}{ Floor } & \multicolumn{2}{c}{ Foam } & \multicolumn{3}{c}{ Old $(\mathbf{n}=\mathbf{2 9})$} \\
\hline & Eyes open & Eyes closed & Eyes open & Eyes closed & Eyes open & Eyes closed & Eyes open & Eyes closed \\
\hline AP displacement & 15.98 & 19.17 & 22.86 & 33.48 & $20.36^{+}$ & $26.09^{*}$ & 25.90 & $46.80^{*}$ \\
& $(5.35)$ & $(8.71)$ & $(10.11)$ & $(13.45)$ & $(6.99)$ & $(10.79)$ & $(7.86)$ & $(19.51)$ \\
\hline ML displacement & 12.61 & 15.64 & 19.83 & 29.17 & $23.08^{*}$ & $27.07^{*}$ & $32.93^{*}$ & $52.02^{*}$ \\
& $(5.24)$ & $(8.54)$ & $(6.31)$ & $(10.64)$ & $(11.50)$ & $(15.43)$ & $(17.79)$ & $(23.42)$ \\
\hline Path length & 59.33 & 83.97 & 108.27 & 195.03 & $106.00^{*}$ & $135.08^{*}$ & $186.54^{*}$ & $359.36^{*}$ \\
& $(25.98)$ & $(34.08)$ & $(46.72)$ & $(51.81)$ & $(44.96)$ & $(62.91)$ & $(74.25)$ & $(169.72)$ \\
\hline
\end{tabular}

*significantly different to Young $(p<0.01)$

${ }^{+}$significantly different to Young $(p<0.05)$

Bonferroni post-hoc tests showing significant differences between all conditions $(\mathrm{p}<0.024)$. The group*condition main effect was not significant $\left(\mathrm{F}_{3,473}=0.13, \mathrm{p}=0.943\right)$.

\section{Swaymeter Convergent Validity}

Table 3 presents Pearson correlation coefficients between Swaymeter and forceplate (COP) data. The Swaymeter measures were moderately to strongly associated with COP measures, with correlation coefficients between 0.560 and 0.865 . In particular, the Swaymeter had excellent correlation with COP for the AP displacement measure, with correlation coefficients greater than 0.74 across all conditions.

Table 3 also presents offsets and limits of agreement between Swaymeter and forceplate (COP) measures. Since Bland-Altman plots were similar across conditions, representative plots are presented in Figure 2. These plots indicate good agreement between Swaymeter and COP data for AP and ML displacement (Figure $2 \mathrm{a}, \mathrm{b})$, since few datapoints fall outside of the limits of agreement (95\% CI). The mean Swaymeter-COP offset for AP displacement indicates increased magnitude recorded from the forceplate system, which was similar across the range of data. Minimal Swaymeter-COP offsets were seen for ML displacement. The sway path length was considerably larger for COP compared to Swaymeter data, with the difference increasing with the magnitude of sway (Figure 2c). Following log-transformation of sway path datasets, the relationship between
Swaymeter and faceplate sway path was consistent across the range of data (Figure $2 \mathrm{~d}$ ).

\section{Repeatability}

ICCs (95\% CIs) and SEMs for Swaymeter data recorded during 3 repeated trials are presented in Table 4. Swaymeter measures showed good-to-excellent repeatability across conditions, with ICCs ranging from 0.654 to 0.944. Path length measures showed particularly high repeatability, with all ICCs in excess of 0.83 .

\section{Discussion}

Control of the whole body COM is the primary goal of the balance system. The Swaymeter provides an indirect measure of COM movement as it is fixed at approximately the level of the COM (pelvis) and records motion of the body in $2 \mathrm{D}$ while standing. In order to stabilise the COM, an individual produces forces on the support surface/s (predominantly under the feet while standing). Due to the relative ease of forceplate recordings, the COP has commonly been used in previous studies of standing balance control as an indicator of balance stability. However, while COM and COP measures are related, they are not synonymous [17]. The COP-COM position separation varies across individuals and conditions. Despite this, the Swaymeter showed good agreement with forceplate COP measures for AP and ML displacement (average offset $=6$ $\mathrm{mm}$ ) and moderate-excellent correlations for AP ( $\mathrm{r}>$ 0.743) and ML displacement ( $\mathrm{r}$ 0.692).

Table 3 Pearson correlation coefficients ( $\mathrm{r}$ ), followed by Bland-Altman offsets $(\mathrm{mm})$ and limits of agreement $(95 \% \mathrm{Cls})$ for sway measures, as determined from Swaymeter recordings, against those taken from forceplate (COP) recordings.

\begin{tabular}{|c|c|c|c|c|}
\hline & \multicolumn{2}{|c|}{ Floor } & \multicolumn{2}{|c|}{ Foam } \\
\hline & Eyes open & Eyes closed & Eyes open & Eyes closed \\
\hline $\begin{array}{l}\text { AP displacement } \\
\text { Offset }(95 \% \mathrm{Cl})\end{array}$ & $\begin{array}{c}r=0.743 \\
4.5(-6.8-15.7)\end{array}$ & $\begin{array}{c}r=0.845 \\
6.8(-3.2-16.7)\end{array}$ & $\begin{array}{c}r=0.820 \\
9.5(-4.9-23.8)\end{array}$ & $\begin{array}{c}r=0.865 \\
15.6(-5.2-36.4)\end{array}$ \\
\hline $\begin{array}{l}\text { ML displacement } \\
\text { Offset }(95 \% \mathrm{Cl})\end{array}$ & $\begin{array}{c}r=0.692 \\
-4.1(-19.5-11.3)\end{array}$ & $\begin{array}{c}r=0.752 \\
-5.4(-25.0-14.2)\end{array}$ & $\begin{array}{c}r=0.733 \\
0.1(-19.0-19.3)\end{array}$ & $\begin{array}{c}r=0.807 \\
-2.7(-30.1-24.7)\end{array}$ \\
\hline $\begin{array}{l}\text { Path length } \\
\text { Offset }(95 \% \mathrm{Cl})\end{array}$ & $\begin{array}{c}r=0.667 \\
231.0(68.6-393.4)\end{array}$ & $\begin{array}{c}r=0.754 \\
275.5(-58.1-609.1)\end{array}$ & $\begin{array}{c}r=0.560 \\
409.2(55.1-763.3)\end{array}$ & $\begin{array}{c}r=0.858 \\
590.0(-363.9-1543.9)\end{array}$ \\
\hline
\end{tabular}




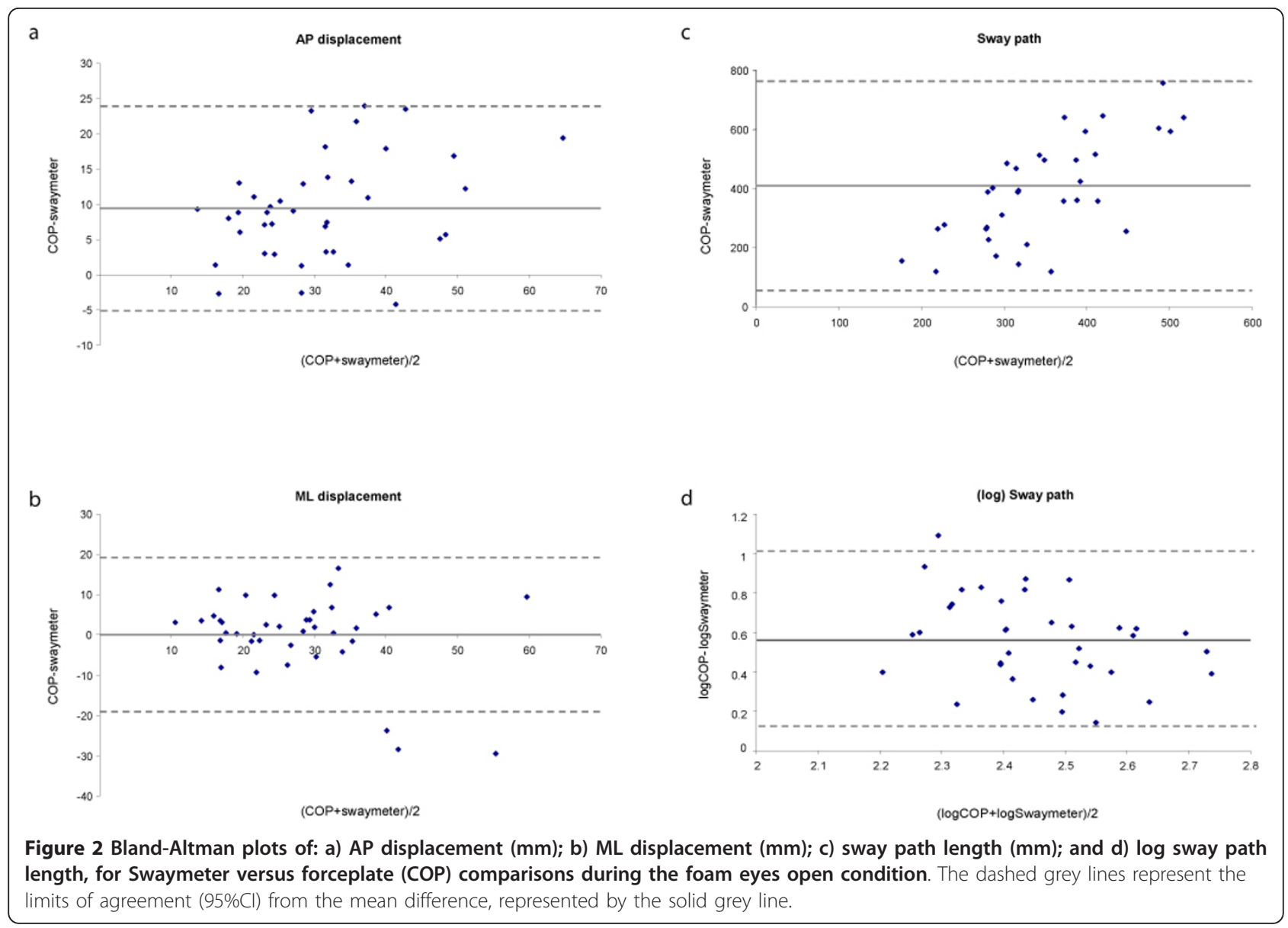

While moderate correlations $(r>0.560)$ were found for Swaymeter versus forceplate sway path length, Bland-Altman plots revealed measures were consistently longer for the forceplate compared to the Swaymeter (average offset $=376 \mathrm{~mm}$ ). Since correlations tend to increase when the data are more widely spread, the Bland-Altman statistics provide more meaningful detail regarding measurement agreement. The Bland-Altman results show that the magnitude of difference between Swaymeter and forceplate measures of sway path increased with the magnitude of sway. This result may be due to the reduced precision of the Swaymeter (1 $\mathrm{mm}$ ), the dampening of body motion through the Swaymeter and pen-paper interface friction, and the higher frequency motions recorded by the forceplate system. Increased filtering of COP data might improve the agreement between these measures. However, these results suggest that postural sway path length cannot be directly compared between Swaymeter and forceplate recordings. Subsequent analyses on log-transformed sway path datasets showed good agreement across the range, indicating that data from these sources may be compared following log-transformation. However, it should be noted that log transformations of Swaymeter

Table 4 Intraclass correlation coefficients (95\% Cls) and Standard Error of Means (SEM) for sway measures across four conditions, as determined from Swaymeter recordings.

\begin{tabular}{|c|c|c|c|c|}
\hline & \multicolumn{2}{|c|}{ Floor } & \multicolumn{2}{|c|}{ Foam } \\
\hline & Eyes Open & Eyes Closed & Eyes Open & Eyes Closed \\
\hline AP displacement & $\begin{array}{c}.654(.414-.806) \\
\text { SEM }=.014\end{array}$ & $\begin{array}{c}.774(.618-.873) \\
\text { SEM }=.017\end{array}$ & $\begin{array}{c}.666(.438-.812) \\
\text { SEM }=.013\end{array}$ & $\begin{array}{c}.777(.616-.877) \\
\text { SEM }=.017\end{array}$ \\
\hline ML displacement & $\begin{array}{c}.763(.597-.867) \\
\text { SEM }=.022\end{array}$ & $\begin{array}{c}.821(.697-.899) \\
\text { SEM = .024 }\end{array}$ & $\begin{array}{c}.792(.650-.883) \\
\text { SEM }=.020\end{array}$ & $\begin{array}{c}.823(.697-.902) \\
\text { SEM }=.021\end{array}$ \\
\hline Path length & $\begin{array}{c}.834(.720-.906) \\
\text { SEM }=.020\end{array}$ & $\begin{array}{c}.944(.906-.969) \\
\text { SEM }=.019\end{array}$ & $\begin{array}{c}.894(.816-.941) \\
\text { SEM }=.019\end{array}$ & $\begin{array}{c}.933(.885-.963) \\
\text { SEM }=.021\end{array}$ \\
\hline
\end{tabular}


data are not required in clinical settings, as raw scores can be contrasted to population norms to provide an indication of an individual's postural stability [16].

Immediate test-retest repeatability was found to be good for all Swaymeter measures across the 4 conditions. The correlation coefficients across trials were excellent for sway path length (ICC > 0.834) and ML displacement (ICC > 0.763), and good for AP displacement (ICC > 0.654). Data from all participants were included in these analyses to cover a fuller range (spread) of data points, as the issue of interest was the device, not the participant group. However, similar associations were evident when the analyses were restricted to the older group alone (data not shown). The superior Swaymeter ICCs in the ML plane, relative to AP, are in agreement with those previously reported for within-day COP velocity ICCs $[18,19]$. Within session reliability is largely related to the random variability of the measurement, as opposed to intersession reliability which may incorporate changes in postural stability over a longer period of time and errors associated with reapplication of setup protocols. Lin and colleagues [18] also found within-day reliability to be better than between-day reliability for both young and older adult groups. Similarly, we found higher reliability coefficients for immediate measures compared with those previously measured for repeats over a longer period [4], which suggests that an individual's postural sway may change over days or weeks.

Sway variables measured with the Swaymeter significantly differed between young and older participants, in line with previous findings [20]. These differences provide evidence of Swaymeter concurrent validity for examining age-related differences in postural sway. One quarter of our older adult group reported a fall in the previous year, suggesting that we recruited a representative sample of healthy community-dwelling older adults living in the Australian state of New South Wales [21]. The between-group differences reported here are, therefore, indicative of normal age-related declines in postural sway in healthy community-dwelling older people.

Maki and colleagues [5] have suggested that a quick, simple, and safe measure of postural sway provides a better prediction of future falling risk in older people compared with measures derived using more complicated and expensive moving platform protocols that induce balance responses. Piirtola and Era [3] reviewed nine prospective studies of forceplate sway measures and found only five to be significantly associated with falls outcomes in older people. In contrast, Swaymeter measures have consistently shown significant associations with future falls across multiple populations of older people (ie people living in both the community and residential care) $[11,12,22]$. The consistent findings most likely relate to portability and feasibility factors, which have allowed assessment of large sample sizes (with resultant increased statistical power) and assessment of older people across a broad stability spectrum (with resultant high inter-participant sway variability). It is also possible that apparent limitations of the Swaymeter, including the cross-talk between ML and yaw motion (twisting) resulting in amplified Swaymeterrecorded ML displacement and sway path length and/or the dampening of pelvis motion through the device and pen-paper interface friction, provide measures that are more predictive of future falls than forceplate COP measures. For example, future fallers may have more yaw motion while standing, which is detected by larger ML displacement from Swaymeter recordings but not seen with COP measures. It should be noted that the Swaymeter is subject to operator error, as sway variables are counted and computed manually.

\section{Conclusions}

The Swaymeter enables measurement of postural sway that is simple and less expensive than methods employing forceplate or motion capture systems. This study has found the Swaymeter to be a reliable measure of postural sway that discriminates between young and older people across multiple sensory conditions. Sway displacement measures from the Swaymeter agreed well with forceplate measures, while sway path measures showed an offset that increased with the magnitude of sway, which can be overcome by correcting the skewed data via log-transformation.

\section{List of abbreviations}

AP: Anteroposterior; Cl: Confidence intervals; COM: Centre of mass; COP: Centre of pressure; ICC: Intraclass correlation coefficients; ML: Mediolateral; SEM: Standard error of the mean; SD: Standard deviation.

\section{Acknowledgements}

Dr Sturnieks was supported by a NHMRC Population Health Capacity Building Grant in Injury Prevention, Acute Care and Rehabilitation. Prof Lord was supported by a NHMRC Fellowship.

\section{Authors' contributions}

DS conceived the study, participated in its design and coordination, collected and processed the data, helped with statistical analyses and drafted the manuscript. RA reviewed literature, helped with statistical analyses and drafted the manuscript. SL participated in the study design and coordination, helped with statistical analyses and drafted the manuscript. All authors have read and approve the final manuscript.

\section{Competing interests}

The Swaymeter is commercially available from Neuroscience Research Australia. The authors declare that they have no other competing interests

Received: 24 January 2011 Accepted: 20 October 2011

Published: 20 October 2011

\section{References}

1. Baloh RW, Fife TD, Zwerling L, Socotch T, Jacobson K, Bell T, Beykirch K: Comparison of static and dynamic posturography in young and older normal people. J Am Geriatr Soc 1994, 42(4):405-12. 
2. Hellbrandt FA, Braun GL: The influence of sex and age on the postural sway of man. Am J Phys Anthropol 1939, 24(3):347-360.

3. Piirtola M, Era P: Force platform measurements as predictors of falls among older people - a review. Gerontology 2006, 52(1):1-16.

4. Lord SR, Castell S: Physical activity program for older persons: effect on balance, strength, neuromuscular control, and reaction time. Arch Phys Med Rehabil 1994, 75(6):648-52.

5. Maki BE, Holliday PJ, Topper AK: A prospective study of postural balance and risk of falling in an ambulatory and independent elderly population. Journal of Gerontology 1994, 49(2):M72-84.

6. Brooke-Wavell K, Prelevic GM, Bakridan C, Ginsburg J: Effects of physica activity and menopausal hormone replacement therapy on postural stability in postmenopausal women-a cross-sectional study. Maturitas 2001, 37(3):167-72.

7. Chang AT, Seale H, Walsh J, Brauer SG: Static balance is affected following an exercise task in chronic obstructive pulmonary disease. J Cardiopulm Rehabil Prev 2008, 28(2):142-5.

8. Hinman RS, Bennell KL, Metcalf BR, Crossley KM: Balance impairments in individuals with symptomatic knee osteoarthritis: a comparison with matched controls using clinical tests. Rheumatology (Oxford) 2002, 41(12):1388-94.

9. Lord SR, McLean D, Stathers G: Physiological factors associated with injurious falls in older people living in the community. Gerontology 1992, 38(6):338-46.

10. Lord SR, Sambrook PN, Gilbert C, Kelly PJ, Nguyen T, Webster IW, Eisman JA: Postural stability, falls and fractures in the elderly: results from the Dubbo Osteoporosis Epidemiology Study. Medical Journal of Australia 1994, 160(11):684-5, 688-91.

11. Lord SR, Clark RD: Simple physiological and clinical tests for the accurate prediction of falling in older people. Gerontology 1996, 42(4):199-203.

12. Lord SR, Ward JA, Williams P, Anstey KJ: Physiological factors associated with falls in older community-dwelling women. J Am Geriatr Soc 1994, 42(10):1110-7

13. Folstein MF, Folstein SE, McHugh PR: "Mini-Mental state": a practical method for grading the cognitive status of patients for the clinician. $J$ Psychiatr Res 1975, 12:189-98.

14. Lord SR, Clark RD, Webster IW: Postural stability and associated physiological factors in a population of aged persons. J Gerontol 1991, 46(3):M69-76.

15. Bland JM, Altman DG: Statistical methods for assessing agreement between two methods of clinical measurement. Lancet 1986, 1(8476):307-10.

16. Lord SR, Menz HB, Tiedemann A: A physiological profile approach to falls risk assessment and prevention. Phys Ther 2003, 83(3):237-52.

17. Winter DA, Patla AE, Prince F, Ishac M, Gielo-Perczak K: Stiffness control of balance in quiet standing. J Neurophysiol 1998, 80(3):1211-21.

18. Lin D, Seol H, Nussbaum MA, Madigan ML: Reliability of COP-based postural sway measures and age-related differences. Gait Posture 2008, 28(2):337-42.

19. Lafond D, Corriveau H, Hebert R, Prince F: Intrasession reliability of center of pressure measures of postural steadiness in healthy elderly people. Arch Phys Med Rehabil 2004, 85(6):896-901.

20. Lord SR, Ward JA: Age-associated differences in sensori-motor function and balance in community dwelling women. Age Ageing 1994, 23(6):452-60

21. Centre for Health Advancement and Centre for Epidemiology and Research: New South Wales Falls Prevention Baseline Survey: 2009 Report. NSW Department of Health Sydney; 2010.

22. Lord SR, Clark RD, Webster IW: Physiological factors associated with falls in an elderly population. Journal of the American Geriatrics Society 1991, 39(12):1194-200.

\section{Pre-publication history}

The pre-publication history for this paper can be accessed here: http://www.biomedcentral.com/1471-2318/11/63/prepub

doi:10.1186/1471-2318-11-63

Cite this article as: Sturnieks et al: Validity and reliability of the Swaymeter device for measuring postural sway. BMC Geriatrics 2011 11:63.

\section{Submit your next manuscript to BioMed Central and take full advantage of:}

- Convenient online submission

- Thorough peer review

- No space constraints or color figure charges

- Immediate publication on acceptance

- Inclusion in PubMed, CAS, Scopus and Google Scholar

- Research which is freely available for redistribution

Submit your manuscript at www.biomedcentral.com/submit 